\title{
Robonaut 2 on the International Space Station: Status Update and Preparations for IVA Mobility
}

Thomas D. Ahlstrom ${ }^{*}$ and Myron A. Diftler ${ }^{\dagger}$ and Reginald B. Berka ${ }^{\ddagger}$ and Julia M. Badger ${ }^{\S}$ and Sandeep Yayathi** NASA Johnson Space Center, Houston, TX, 77058

Andrew W. Curtis ${ }^{\dagger \dagger}$ and Charles A. Joyce ${ }^{\dagger+}$

Oceaneering Space Systems, Houston, TX, 77058

\begin{abstract}
Robotics engineers, ground controllers and International Space Station (ISS) crew have been running successful experiments using Robonaut 2 (R2) on-board the ISS for more than a year. This humanoid upper body robot continues to expand its list of achievements and its capabilities to safely demonstrate maintenance and servicing tasks while working alongside human crewmembers. The next phase of the ISS R2 project will transition from a stationary Intra Vehicular Activity (IVA) upper body using a power/data umbilical, to an IVA mobile system with legs for repositioning, a battery backpack power supply, and wireless communications. These upgrades will enable the $R 2$ team to evaluate hardware performance and to develop additional control algorithms and control verification techniques with $\mathbf{R 2}$ inside the ISS in preparation for the Extra Vehicular Activity (EVA) phase of R2 operations. As R2 becomes more capable in assisting with maintenance tasks, with minimal supervision, including repositioning itself to different work sites, the ISS crew will be burdened with fewer maintenance chores, leaving them more time to conduct other activities. R2's developers at the Johnson Space Center (JSC) are preparing the R2 IVA mobility hardware and software upgrades for delivery to the ISS in late 2013. This paper summarizes R2 ISS achievements to date, briefly describes the R2 IVA mobility upgrades, and discusses the R2 IVA mobility objectives and plans.
\end{abstract}

\footnotetext{
* Robonaut Flight Project Manager, Crew and Thermal Systems Division, 2101 NASA Parkway, Houston, TX 77058, Mail Stop: EC7, AIAA Member Grade

${ }^{\dagger}$ Robonaut Project Manager, Software Robotics and Simulation Division, 2101 NASA Parkway, Houston, TX 77058, Mail Stop: ER4, AIAA Member Grade

* Robonaut Engineer, Software Robotics and Simulation Division, 2101 NASA Parkway, Houston, TX 77058, Mail Stop: ER4, AIAA Member Grade

$\S$ Robonaut Project Software and Applications Lead, Software Robotics and Simulation Division, 2101 NASA Parkway, Houston, TX 77058, Mail Stop: ER4, AIAA Member Grade

** Robonaut Project Backpack Development Lead, Software Robotics and Simulation Division, 2101 NASA Parkway, Houston, TX 77058, Mail Stop: ER4, AIAA Member Grade.

${ }^{\dagger}$ Robonaut Project Leg Development Lead, Software Robotics and Simulation Division, 2101 NASA Parkway, Houston, TX 77058, Mail Stop: ER4, AIAA Member Grade.

$\$$ Robonaut Project Deputy Manager, Software Robotics and Simulation Division, 2101 NASA Parkway, Houston, TX 77058, Mail Stop: ER4, AIAA Member Grade
} 


\section{Introduction}

$\mathrm{R}$ OBOTIC systems in Low Earth Orbit (LEO) have established an impressive record over the past several decades. The most famous, the Shuttle Remote Manipulator System (SRMS) has manipulated a large number of payloads and played a key role in the assembly of the International Space Station. Similarly the Space Station Remote Manipulator System (SSRMS) not only participated in the International Space Station (ISS) assembly, it continues to serve a vital role in managing the vehicles that visit the station and in space station maintenance activities. ${ }^{1}$ Both of these large robotic systems have proven their value by reliably moving large payloads and the astronauts in support of Extravehicular Activities (EVA).

In contrast to the large space robots, several smaller systems have been deployed over the past 20 years. ROTEX, developed by the German Space Agency, flew on STS-55 in 1993, and served as the first ground-controlled robot in space. ${ }^{2}$ The National Space Development Agency of Japan (NASDA) launched the Engineering Test Satellite (ETS) VII in 1997. ${ }^{3}$ Among the experiments performed on the satellite were a number of robotic tests, including remote operation from the ground, coordinated control of the robot arm, and Orbital Replacement Unit (ORU) change out. Also in 1997, a NASA robot, AERCam-Sprint, flew on STS-87. ${ }^{4}$ AERCam is a powered free flyer that gives crewmembers the ability to obtain camera views from difficult to reach locations in the Extravehicular (EV) environment. During this Shuttle flight experiment, the crew

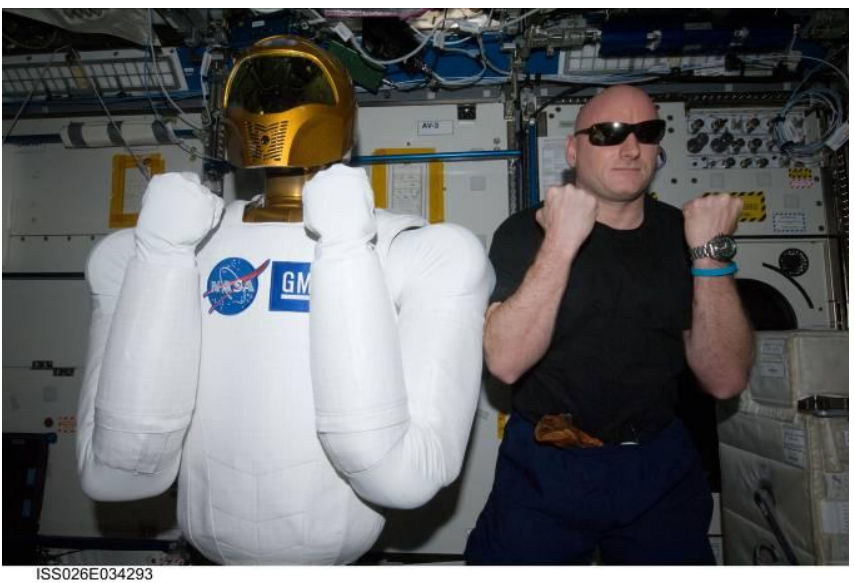

Figure 1. R2 on ISS along with Astronaut Scott Kelly remotely controlled this robot showing its utility for collecting unique and very valuable video. More recently, the German Space Agency positioned a two joint robotic arm on the outside of the ISS Russian Module to demonstrate a variety of control modes and gather data on the long term performance of the system's modular joints. ${ }^{5}$ All of these experiments have significantly advanced the state-of-the-art of space robots and demonstrated the ability for remote robots to perform meaningful work.

The impetus behind these demonstrations was to develop robotic capabilities that will help crew and ground controllers maintain ISS equipment located on the outside of the vehicle. ISS currently has one system on-board specifically designed to perform this function. The Canadian Space Agency's Special Purpose Dexterous Manipulator $(\mathrm{SPDM})^{1}$ has successfully completed its initial servicing activities on equipment with EVA robotically compatible interfaces. It also has successfully tested several new robotically compatible tools. ${ }^{6}$ However, currently no robotic system on ISS is certified to assist with EVA tasks that are currently only performed by crew. This is Robonaut's future role.

Robonaut is designed to setup and tear down EVA work sites, saving as much as 90 minutes on either side of a task. It can work side by side with a human, hand off tools and other humanly compatible equipment and free up crew time for more valuable activities. Activated on-board ISS in August, 2011, the latest version of Robonaut, Robonaut 2, also known as R2, is an anthropomorphic system designed to perform these tasks. As part of a carefully orchestrated series of tests and upgrades, R2 is currently performing tasks while mounted to a fixed based stanchion, and it will soon be augmented with legs to obtain Intravehicular (IV) mobility allowing it to assist crew inside the station. A future set of upgrades will convert it to an EV system where it will have its greatest payoff in terms of assisting crew.

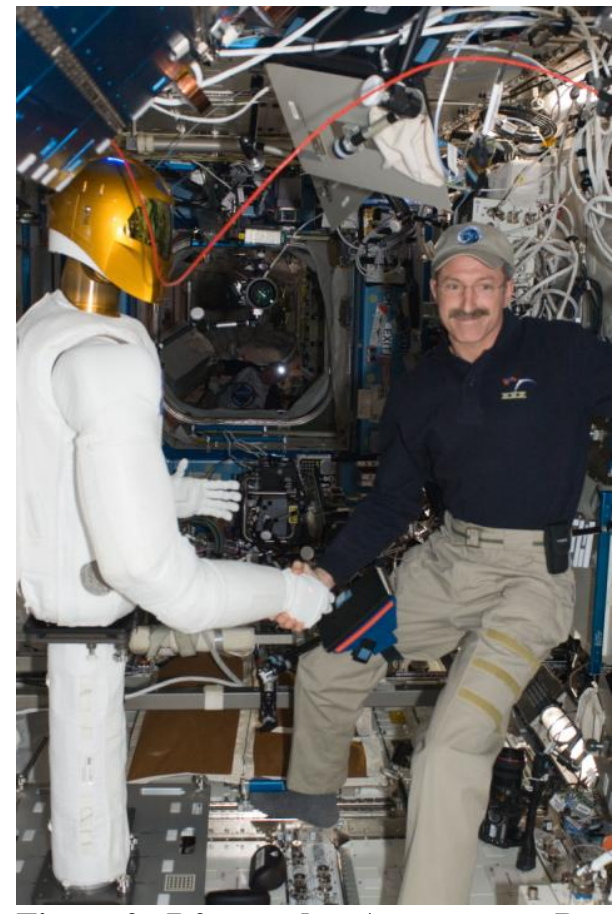

Figure 2. R2 and Astronaut Dan Burbank share the first humanoid robotic handshake in space 


\section{Robonaut 2 (R2) Overview}

The Robonaut 2 upper body is a state of the art, dexterous, anthropomorphic robot with significant technical improvements over its predecessor, making it a far more valuable tool for astronauts. ${ }^{7}$ Upgrades include: increased force sensing, greater range of motion, higher bandwidth, and improved dexterity. R2's integrated mechatronic design results in a more compact and robust distributed control system with a fraction of the wiring of the original Robonaut. Modularity is prevalent throughout the hardware and software along with innovative and layered approaches for sensing and control. The most important aspects of the Robonaut philosophy are clearly present in this latest model's ability to allow comfortable human interaction and in its design to perform significant work using the same hardware and interfaces used by people.

With 42 independent degrees-of-freedom (DOF's) and over 350 sensors, Robonaut 2 is an impressive example of mechatronic integration. Encompassing two 7-DOF arms, two 12-DOF hands, a 3-DOF neck and a single DOF waist, the system includes 50 actuators with collocated, low-level joint controllers embedded throughout the robot. The system also integrates built-in computing and power conversion inside its backpack and torso.

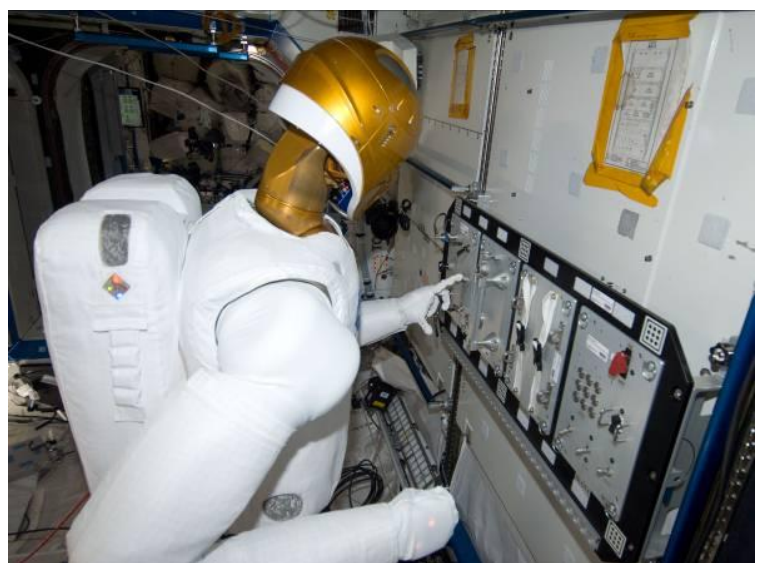

Figure 3. Robonaut 2 (R2) onboard ISS working with its task board.

Robonaut 2 uses brushless DC motors, harmonic drive gear reductions, and electromagnetic failsafe brakes as the building blocks for the power and torque dense actuators in the robot's human-scale, upper arms. The use of series elastic actuation, however, differentiates R2 from R1. This technology integrates springs into the joint transmission allowing for many important benefits, including shock tolerance, energy storage and additional options for achieving high resolution force control. Developed initially for legged robots, series elastic actuators (SEA's) are now being found in humanoid robot arms where the advantages are being explored and utilized. ${ }^{8}$

The Robonaut 2 hand $^{9}$ and forearm are designed to approximate human hand capabilities. The five fingered, 12 DOF hand and the forearm, which houses two wrist degrees-of-freedom, is a modular, extremely dexterous, standalone end-effector. The Robonaut 2 thumb has four joints allowing for a wider range of grasps. The thumb, working with the primary fingers, achieves a range of dexterous grasps. When combined with the ring and pinkie finger, the $\mathrm{R} 2$ hand can form various power and tool grasps. The hand has the capability to manipulate a large set of EVA tools, conventional hand tools, and soft goods.

\section{R2 ISS Achievements to Date}

The Robonaut Team took a step by step approach to activating and beginning motion operations onboard ISS. Great lengths were taken to protect $\mathrm{R} 2$ on the ride to ISS; however, a thorough inspection and checkout program were followed to ensure that R2 was functioning properly prior to expanding operations.

Before free space or contact operations began, checkouts of R2's safety systems were performed. This involved the on-orbit crew removing R2 from its launch packaging and assembling it onto its support equipment which launched alongside the robot. R2's many processors and sensors were checked for functionality to ensure that nothing was damaged during the Space Shuttle launch. Each of R2's joints were checked for proper functionality and that the torque measurements within each joint were reporting to the brainstem processors as expected. Six checkout operations verified R2's system health prior to Robonaut

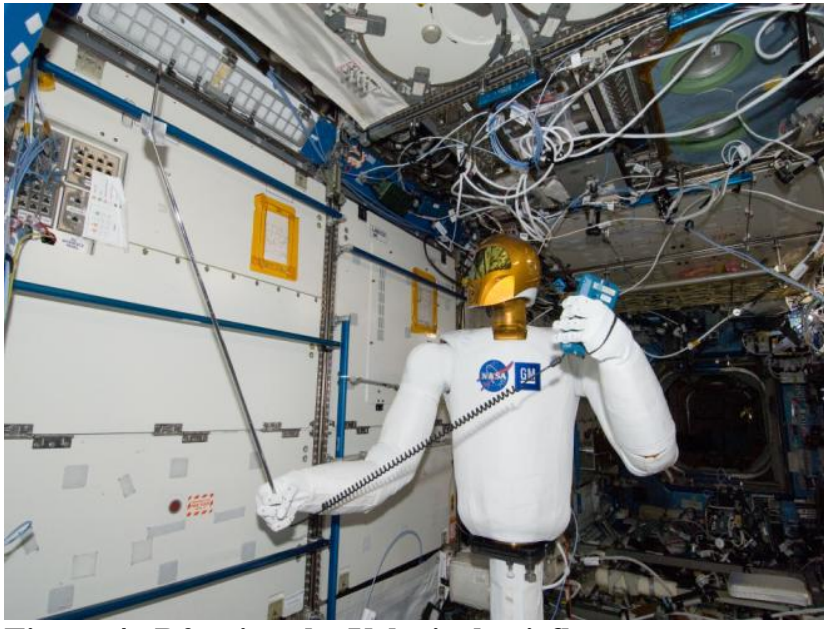

Figure 4. $\mathrm{R} 2$ using the Velocicalc airflow meter performing larger scale free space operations. 
Once the R2 team, and the NASA safety community, were satisfied that all of R2's safety systems were functioning properly, free space and contact operations commenced. Free space operations involve multiple joint motions simultaneously. Contact operations are performed with the task board that was also flown to ISS along with R2. The task board has a modular design with multiple task panels that can be interchanged to provide flexibility in the tasks that R2 performs on-orbit. The powered panel contains an array of push buttons, two and three-way switches, a rocker switch with finger guards and a power switch with a guard that covers the entire switch. The unpowered panel supplies various valves and connectors that can be found on ISS hardware. An EVA task panel provides an EVA handrail, a tether point and various EVA fittings that are commonly used for handling hardware by spacewalkers. A stowage panel was flown with various restraining options so ISS hardware could be attached to the panel for R2 experiments. Finally, the softgoods panel provides a large box covered in softgoods that R2 can manipulate. ${ }^{10}$ Softgoods are very common both inside and outside ISS for storing and protecting hardware. This task board and its task panels have been used many times by R2 during onorbit operations to practice and improve the motion scripts for performing generic tasks such as flipping switches, cleaning handrails and manipulating softgoods.

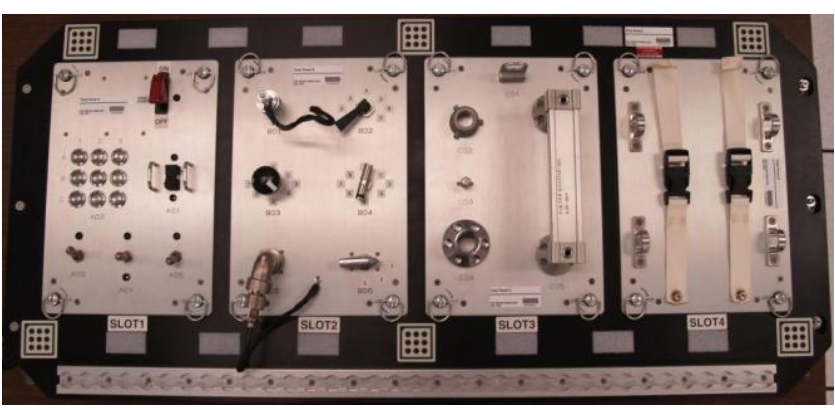

Figure 5. R2's task board

The task board has also been used to test R2's machine vision. This new vision recognition system can identify objects based on their shape and color and allow R2 to autonomously identify objects. It also allows R2 to autonomously verify if a switch or lever has been successfully toggled or not. If R2 attempts to flip a switch, it can self-verify if this was successful or not by using machine vision. If it was unsuccessful, R2 will try again with small modifications to the script to increase the odds of success. This machine vision system will continue to be developed and used in the stationary configuration and will be a vital tool for mobility operations to come.

There are a limited number of tasks available on the task board, so Robonaut is beginning to use tools and venture beyond the task board and into other ISS activities. The first tool operation that was performed was with an air flow meter called Velocicalc. This tool is used to measure the air flow rate coming out of the air vents onboard ISS. Depending on the rate at which air is coming out of the vent, ground controllers and engineers can determine if the air filters need to be changed out. This is a tedious task for the crew to perform. The Velocicalc has to be held very steady to get an accurate reading and the crew has to call down the air flow rate real-time to the ground controllers. This air flow rate jumps around quickly so reading down an accurate measurement can be difficult. However, R2 is perfectly designed for these types of tasks. R2 can remain very steady while taking a measurement and can also use its vision system to relay the actual air flow reading off of Velocicalc real-time to the ground controllers. R2 has performed this task twice already and plans are to continue to support the Environmental Control and Life Support System (ECLSS) Team with similar tasks in the future.

Thus far, R2 has most often been operated from the ground by ground controllers. However, there is a second operating mode that can provide increased flexibility in the tasks R2 can perform. This operating mode is called teleoperation. This operating mode involves an on-orbit crew member donning a vest, gloves and a virtual reality (VR) helmet. A tracker bar is attached to ISS structure in front of the crew member that monitors the motion of the crewmember. These movements are then sent to the robot and R2 mimics the crewmember's motion. This allows astronauts to make real time decisions that R2 will follow. Astronaut Tom Marshburn performed R2's first teleoperation task on-orbit in April of 2013. R2 and the Robonaut team will continue to use this operating mode, as well as ground operation, to continue to demonstrate and expand the number of tasks R2 can perform on board the ISS.

In coming months, Robonaut 2 will begin to experiment with EVA tools on-orbit. EVA tools are specifically designed for use by crewmembers wearing the EVA Mobility Unit (EMU), otherwise known as a

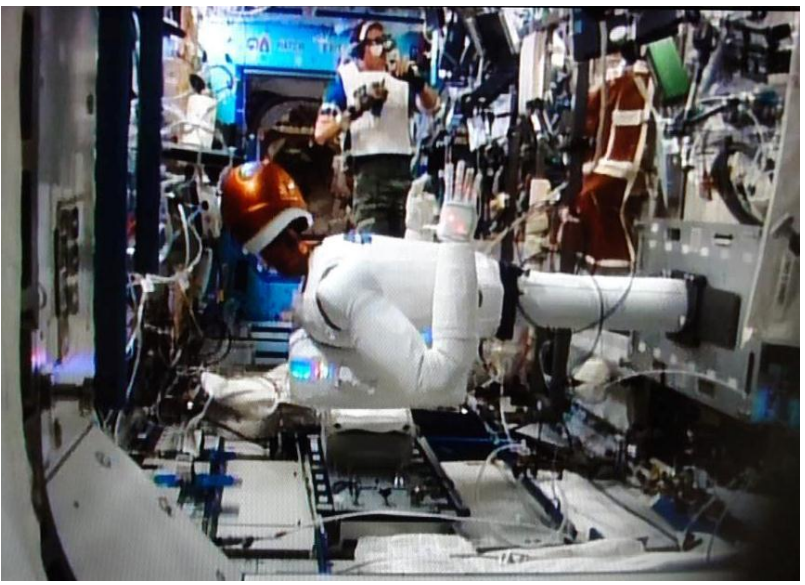

Figure 6. R2 and Astronaut Tom Marshburn performing the first $\mathbf{R} 2$ teleoperation task on ISS 
spacesuit. The EMU has large pressurized gloves that make using tools difficult for spacewalkers. Because of that, many EVA tools are unique and very different than their terrestrial counterparts. Tethers are also a very important part of performing EVAs because floating tools and debris can cause damage to critical ISS systems such as the solar arrays. R2 plans call for tests with both tethers and various tools when interfacing with the EVA task panel.

\section{IVA Mobility Hardware Overview}

The R2 Mobility Hardware includes the Climbing Legs, Battery Backpack and a kit of Mobility Parts that are required for various upgrades to the Torso that is already on-orbit. The Robonaut Team is scheduled to launch this hardware in late 2013 to support on-orbit upgrades and assembly operations that the crew will perform in early 2014.

\section{A. Climbing Legs}

The new R2 Climbing Legs provide locomotion to reposition R2 both inside and outside the ISS and to provide a stable base for torso operations. While their initial qualification is for IVA, they have been designed and built using suitable materials, electrical components, and design features to perform in the EV environment. Each four foot long leg consists of seven series elastic joints arranged in an alternating pitch-roll sequence. Each roll joint has a full 360 degree range of motion and each pitch joint has 160 degrees of range. This kinematic arrangement enables a wide range of stances, poses, and stepping gaits with sufficient reach to traverse both IVA and EVA handrails and the kinematic redundancy to optimize trajectories for factors such as avoiding singularities and obstacles.

The R2 Climbing Leg joints are an evolution of the series elastic R2 arm joints with design enhancements to improve performance and robustness. Key changes include: redesigned motor driver electronics, upgraded sensors, improved component alignment, removal of motor brakes, and a greater use of design modularity.

The motor driver electronics redesign was driven by the requirement to ensure safety, the goal to achieve greater modularity, and the need to adapt to sensor upgrades. While maintaining the same brushless motor driver commutation functionality, the components were repackaged on two stacked Printed Circuit Boards (PCBs) to reduce the overall footprint, separating the Field-Programmable Gate Array (FPGA) and processor functions to different physical chips. This improves fault tolerance, allows for dual channel redundant serial bus communications, and alters the input/output (I/O) capabilities in line with the joint sensor changes.

Repackaging the components on stacked power and logic boards provides a single footprint and volumetric profile that is integrated into all of the R2 Climbing Leg joints, as well as the End Effectors. With a common design, the logistics of manufacturing and sparing are simplified. It also enables multiple design iterations of the power board without requiring replacement of the logic board with its FPGA and processor chips. To achieve acceptable performance for the EVA radiation environment, the Actel ProAsic3 flash based FPGAs and Texas Instruments TMS570 processors with lock-step processing and error detection and correction were selected. All R2 Climbing Leg electrical

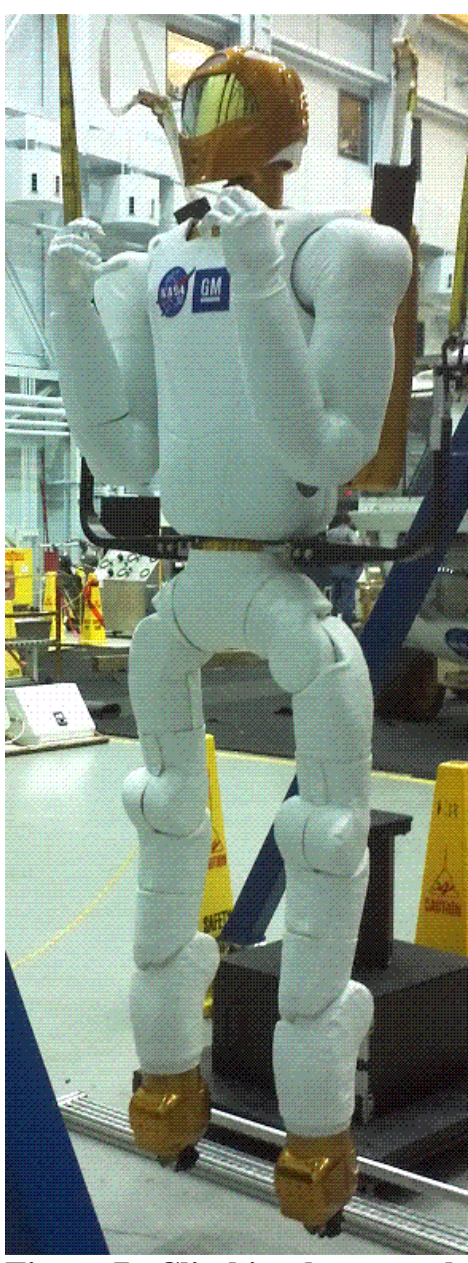

Figure 7. Climbing legs attached to Torso components have undergone radiation testing to verify that radiation-induced events (e.g. bit flips) are detectable, result in non-hazardous configurations, and are non-permanent. Dual serial communications is a new feature supported by the R2 Climbing Leg motor drivers. One channel is designated as the active channel and provides bidirectional communications between the motor drivers and the primary control computer, located in the robot's brainstem, while the second channel is designated passive and provides one-way communication of all data to the secondary brainstem control computer. This redundancy not only checks for corrupt transmissions, but also enables independent cross checking of all coordinated control calculations. The most significant I/O modification to the motor driver was the elimination of the 16 bit Analog to Digital (A/D) capability no longer needed now that the Absolute Position (angle) Sensors (APS) provide digital output. 
Upgraded sensors from the original R2 joint design include using a newly available version of the Netzer rotary Electric Encoders (APSs) with built in A/D calibration and conversion. The motor phase sensors were also replaced with ones less susceptible to magnetic field interference as well as the addition of a bus voltage sensor. The original Mylar optical encoder code disk was also replaced with a metal disk that is able to survive a much greater temperature range.

Improved component alignment in the R2 Climbing Legs assembly was accomplished following analyses of the manufacturing and assembly challenges of the R2 arm joints, resulting in design modifications to achieve the improved fits over the desired temperature range. Examples include bearing interfaces, APS mounting interfaces, and the interfaces between the spring and the joint hardware, which need to be as rigid as possible to prevent nonlinear hysteresis-like spring deflection measurements near zero deflection.

Electromagnetic brakes are not used in the R2 Climbing Leg joints to improve overall on-orbit performance. The electromagnetic brakes require significant power to keep them released during R2 Climbing Leg operations, which is an unnecessary load on the battery. The joint motors can immobilize the joint when needed to oppose large loads and the joints have significant resistance to back drive through the 160:1 harmonic drive trains. As a safety consideration, the ISS crew will also be able to overcome this back drive resistance to move R2 out of the way during an emergency, while they would not have been able to manually overpower the electromagnetic brakes. From an integrated design perspective, not using these brakes also removes a source of electromagnetic emissions near each motor driver and increases the volume for electrical cable routing through the center of the joints.

In addition to using common motor driver electronics for all joints, the R2 Climbing Legs mechanical subassemblies also leverage modularity to reduce unique part count and allow for larger production runs. For example, 12 of the 14 joint motor sub-assemblies are identical and each leg is assembled from three pitch-roll subassemblies. Two of these are identical and the third differs only at its interface with the hip subassembly, which is larger and oriented differently than an adjacent pitch-roll subassembly.

\section{B. End Effectors}

The R2 Climbing Leg End Effectors provide a rigid interface between R2 and the ISS structure by clamping on to handrails or interfacing directly with the seat track rails inside the ISS modules. The End Effector consists of cameras that may be used to conduct visual inspections. As with the rest of the R2 Climbing Legs, the End Effector is qualified for IVA activities, but is designed to eventually be used in the EV environment.

Mechanically, the End Effector uses a roller screw driven 5-bar linkage to open, close, and lock two opposing jaws on the handrail or seat track. When locked, the linkage is in an over center configuration that prevents back driving and opening the jaws. This maintains a firm grasp while the End Effector motor power is turned off. The jaw geometry consists of a cavity shaped to securely grasp the ISS handrails, and cylindrical, undercut studs protruding from the ends of each jaw to engage the mating design features of the seat track. As a backup to motor controlled actuation, the End Effector includes a manual release mechanism on one side that provides a secondary lever to back drive the mechanism out of the locked position.

Electronically, the End Effector uses the same motor driver electronics subassembly as the rest of the Climbing Legs, augmented by two additional sensor interface boards for its unique sensors. For controlling the motor, the End Effector measures the motor angle using an optical encoder, motor currents using phase current sensors, and jaw angle using magnetic APSs located at the pivot of each jaw. For sensing loads, it uses single axis button load cells to measure the clamping force on each of the two jaws. It is mounted to the end of the Climbing Legs through a 6-axis load cell that measures the loads being transferred between the ISS and R2. For sensing proximity to a handrail or seat track while taking steps, the End Effector includes a progressive scan CCD camera and a Photonic Mixer Device (PMD) based 3-Dimensional imaging sensor. These sensors may also be used to conduct close-up visual inspections as needed.

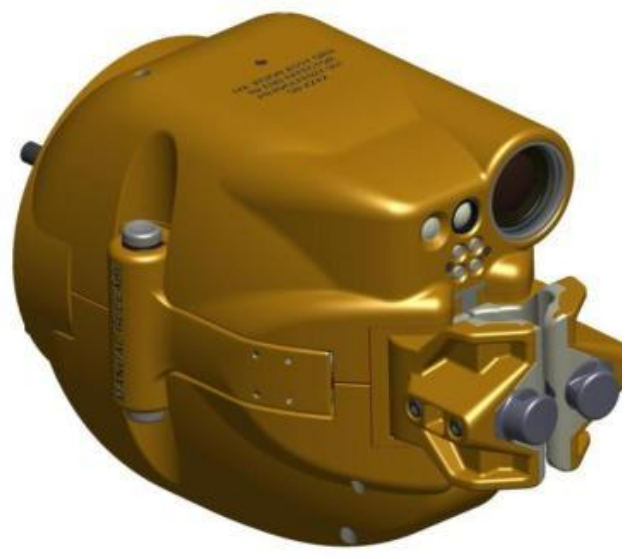

Figure 8. R2 Leg End Effector. 


\section{Battery Backpack}

The existing R2 backpack serves to convert the ISS 120V DC into the requisite $24 \mathrm{~V}, 48 \mathrm{~V}$, and $96 \mathrm{~V}$ bus voltages providing logic and motor power to the robot. The R2 Battery Backpack, nicknamed the Batpack, is designed to facilitate R2 mobility for the duration of an average astronaut EVA. The addition of a large, high energy density battery is necessary to accomplish this goal. In addition, the new Batpack is required to replicate existing functionality with the addition of high energy density batteries, integrated charging capability, and wireless communication.

A lithium-ion based chemistry cell from Boston Power was chosen as the building block of the R2 Battery. Lithium-ion cells offer excellent volumetric and gravimetric energy density. This characteristic allows the Batpack to meet energy, weight, and volumetric requirements. However, while this chemistry offers many technical advantages, it must be maintained and monitored with great care to ensure a safe system. A parallel/series combination of these cells is used to construct the full capacity and stack voltage to operate R2.

Monitoring and safety is accomplished by a tiered architecture including individual slave boards that pass an interlock signal to a master board. The interlock signal is tripped by any slave card when a voltage or temperature limit

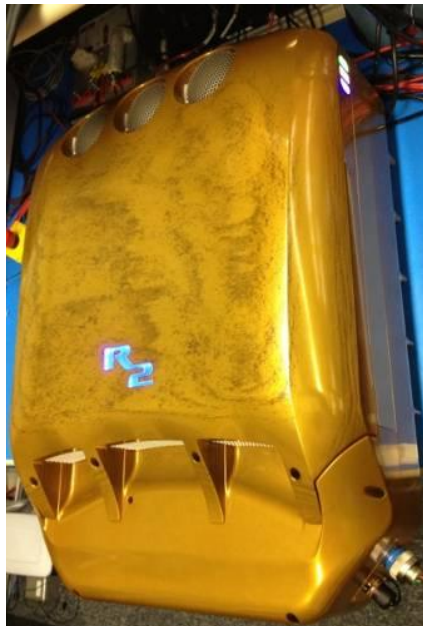

Figure 9. R2 Battery Backpack. is exceeded. The master disconnects the battery from the system when the interlock is broken. The master also controls an integrated battery charger which allows for the pack to be re-charged after it is depleted.

A small embedded device with a Wi-Fi access point satisfies multiple requirements in the system. This unit provides a wireless gateway for R2 communication to the off board control Graphical User Interface (GUI). In addition this device communicates with the master board to log battery data.

The power distribution board converts the battery voltage into the $24 \mathrm{~V}$ and $48 \mathrm{~V}$ buses needed to operate the robot. The original $96 \mathrm{~V}$ bus present in the umbilical backpack will now be powered directly from the battery stack voltage to reduce unnecessary conversion losses. Additional current and voltage sensing is also performed by this component.

The battery cells are packaged in a modular cartridge design with a slave board as well as custom battery management

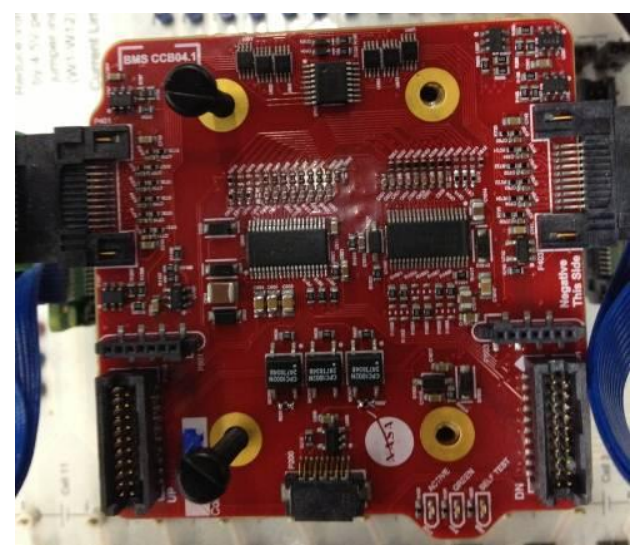

Figure 10. Battery Management Slave Board. electronics to operate and safe the system. The battery cartridge (Fig. 11) contains 60 Boston Power Swing 5300 Lithium-Ion cells. This design allows for different series and parallel combinations of cells for various applications. In the R2 battery these cartridges are configured with five 12-cell virtual cells (cells configured in parallel). The full R2 battery is constructed with 5 of these 60 cell cartridges all connected in series yielding 25 virtual cells in series with each other (Fig. 12). This 300 cell battery pack provides approximately $5.79 \mathrm{kWh}$.

Each cartridge is combined with one battery management slave board that monitors 5 series cells each. This board is also responsible for measuring 12 temperatures at different locations on the cartridge. Each board contains two analog over voltage and under voltage comparators and a hardware temperature comparator. These are all capable of breaking the interlock signal to the master board, resulting in safing of the system. In addition, a special monitoring chip, combined with an analog to digital converter, communicates all of these values to the controller on the master board.

The battery management master board communicates with the slave cards to collect voltage and temperature data. It also generates and receives the interlock signal that is passed through the slave chain. Additional hardware over current, over voltage, and under voltage monitors can break the same interlock signal on the master board itself. If the interlock signal is broken or the master board software determines a limit has been breached, the battery connection to the rest of the system is latched off. A minimum of three redundant

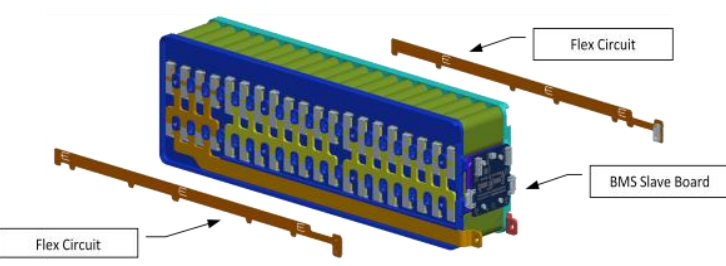

Figure 11. 60 Cell Battery Cartridge. 
mechanisms are in place to safe the battery for each possible hazard condition.

The control loop for the charger electronics in the R2 Batpack is governed by the master board microcontroller. This charger takes in $120 \mathrm{~V}$ DC from the International Space Station and generates the Constant Current - Constant Voltage (CC-CV) profile required for charging this battery safely. Integrating the charger as part of the Batpack system will allow the robot to charge at various Utility Outlet Panel (UOP) locations around the space station. The charger also functions as a power supply to either charge while operating from a fixed location or run the robot entirely with the battery disconnected from the system.

An embedded device containing flash memory, multiple wired Ethernet ports, digital I/O, and a wireless Ethernet adapter is another major component of the Batpack. This module also

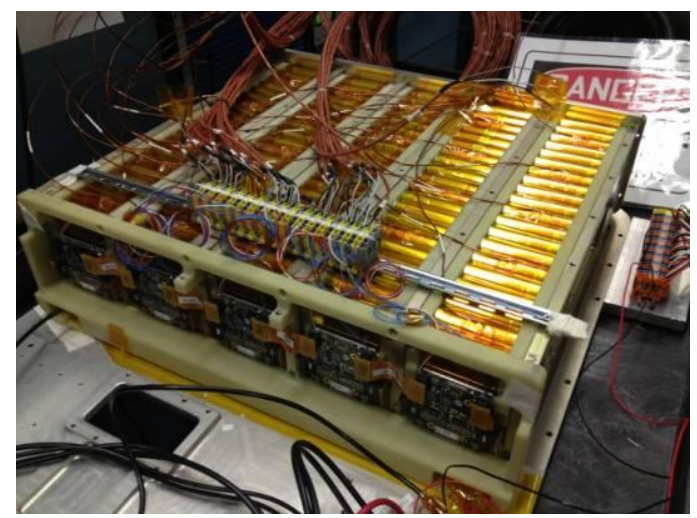

Figure 12. 300 Cell R2 Battery Testing. communicates with the master board to log and store long term battery data. When this device is active, it is possible to continuously track battery trending data while in standby or charging. This allows for download and analysis without requiring the entire robot to be powered on and draining the battery at a higher rate. Finally, this computer also allows wireless power up and power down of the robot without crew interaction. All of these features are necessary to enable Robonaut to be an effective assistant to its human counterparts.

\section{Torso Upgrades}

The existing on-orbit R2 torso was built prior to the development of the Climbing Legs and Batpack and thus will require upgrades to interface with and use them. This torso is suitable for IVA use only and will be replaced by a new torso, currently under development, for EVA operations. The torso upgrades include new computer brainstem components, new interfacing components, and an Inertial Measurement Unit (IMU). To support the additional communications and computation requirements, the brainstem control electronics will be augmented from two complementary computers and one 4-channel I/O board to three independent computers and two 12-channel I/O boards. Additionally, a second 4-channel Ethernet hub and a 4-channel USB hub will be added to the torso. To physically integrate the Climbing Legs with the Torso, a closeout plate will be supplied that the on-orbit crew will pre-install on the bottom of the Torso. The close out plate includes a blind mate connector for making the electrical connections to the Climbing Legs, umbilicals that feed up through the center of the waist joint to connect to the brainstem components (located in R2's chest), and a replacement waist PCB, called a T-Board, with a new connector for routing power and serial data past the waist joint to the Legs. The Batpack is designed to interface with the Torso using the same interface as the existing umbilical backpack. Finally, the torso upgrades include a 6axis IMU to sense accelerations of the torso so that the control system can track and compensate for inertial loads as it moves throughout the ISS.

\section{E. Overview of IVA Mobility Hardware Checkout}

During on-orbit hardware assembly, periodic powered operations will be performed so the R2 Team on the ground can assess if R2 is functioning as expected. The Torso upgrades will be made first, followed by the Climbing Legs and finally the Batpack. A system check will be performed between each step to verify the components are properly installed and functioning as expected. Once the Torso has been upgraded and the Climbing Legs and Batpack assembly are complete, the Robonaut Team will perform verification checks from the ground. Proper communication between the brainstem processors and the limb motor drivers will be confirmed during this checkout and a methodical checkout of the Safety systems will ensure the robot is functioning properly. Once these checks are complete, R2 will be ready for its first ISS Mobility operations.

\section{Safe Operation on ISS}

\section{A. Stationary R2 Safety Background}

The Stationary R2, currently on-orbit, went through a rigorous safety review process prior to its launch on STS133. This review process involved numerous board meetings with the JSC Payload Safety Review Panel (PSRP), extensive testing and analysis of Robonaut's safety systems, and detailed documentation to verify that all necessary requirements were met $^{10}$. 
A Fail Safe Fault Containment Region (FCR) approach was taken to ensure the safety of R2. This approach involves proving that completely separate and independent methods for failing-safe in the event of a hazard exist. An example of a hazard on ISS is inadvertent contact, which results in dynamic loads being transferred into station structure or crewmembers. Two separate FCRs to control this hazard for the stationary R2 include the use of force sensors located in R2's shoulders and the individual joint APSs that measure torque in each joint. Testing was done to verify that these two FCRs are completely independent and, that if one fails, the other is still fully functional. ${ }^{10}$.

Another important aspect of the Robonaut safety system, both for the current on-orbit Torso and future mobility upgrades, is that they are fully functional without a data connection to a computer or processor outside R2's body. It is of vital importance to prove that R2's safety systems are functioning anytime the robot is powered on, even if a connection to a laptop is interrupted, or a wireless signal is lost after R2's mobility upgrades. This is achieved with brainstem processors that are housed inside R2's chest and do not depend on any calculations or data transfer to or from outside processors.

\section{B. On-Orbit Software Updates and Expansion of Capabilities.}

Operating R2 from the ground while R2 itself is onboard ISS, presents an additional set of challenges. Multiple software updates have been performed to incorporate improvements that are realized while performing on-orbit operations. Updates have been made to provide additional data for the user on the ground to monitor. This data provides insight into the health of the robot during operations.

Ground software is also important to the function of R2. Previously, system commands sent to R2 required verification from a secondary ground controller against a previously approved commands list. This was a tedious task and something that was fixed with the development of a new software application called Sentinel. This application automates the command verification process, providing real time checking against approved commands and removing the human-in-the-loop verification. This allows for more efficient and safe operations from the ground.

R2's teleoperation mode, as discussed in section III, required a software update to improve performance. This performance enhancement was achieved via a software update post launch and allowed for smoother control and better trajectory generation allowing R2 to better track the astronauts motions while wearing the teleoperation gear.

Further safety approvals were required for R2 to use tools or equipment after launch and initial deployment on ISS. One of the concerns addressed was the need to tether tools to avoid free floating tools that may escape R2's grasp. By taking a systematic, methodical approach, R2 proved its capability by performing on-orbit operations with the task board and subsequently gained confidence within the safety community regarding Robonaut's safety system. A software update was performed to provide an optional tool-mode where the R2 arms move at a slower velocity to ensure that if a tool is released or inadvertent contact is made, it does not create a safety concern. This update allowed R2 to perform tasks like the Velocicalc air flow task that was described in Section III.

For all software updates, extensive testing is performed. Any time code on the robot is changed, a full suite of safety testing is performed to ensure that none of the FCRs are impacted by the code changes. These software updates and testing results are discussed with the PSRP prior to any software update being implemented.

\section{IVA Mobility Safety Requirements}

As R2 prepares to gain Climbing Legs and a Batpack that will allow it to go mobile inside the ISS, the number of safety requirements and impacts grow. When $\mathrm{R} 2$ is stationary there is a limited amount of surrounding hardware that it can impact. However, when R2 is mobile, the number of potential impacts and interactions between R2 and surrounding hardware grows substantially.

Many of the same requirements apply for a stationary and mobile R2, such as environmental impacts. To ensure the health and safety of the crew, on-orbit off gassing and flammability testing are performed on most non-metallic materials. A database of off gassing and flammability information is kept by the JSC materials team, but any new material not included in the database needs to be tested. The stationary R2 has Lycra covering both the shoulders and elbows, where a material with good stretch characteristics is needed. This material has undesirable flammability characteristics, but it was accepted for the stationary R2 because it is housed in a non-flammable bag when not in use. When R2 is mobile, storing itself in a bag is no longer a good solution. A 100\% Nomex stretch material was found that is non-flammable to replace this Lycra. These shoulder and elbow softgoods pieces will be launched along with the rest of the IVA Mobility Hardware and will be installed by the crew on-orbit. 
There are some new safety requirements and aspects that apply to a mobile R2 that aren't a concern for a stationary R2. Many of the additional requirements and concerns that apply to the mobile system are related to the dynamics of moving hardware like R2. For the stationary R2, the baseplate provides four attachment points to the seat track that are available on the ISS rack faces. This provides a wide and stable base for the stationary robot. The Climbing Legs and End Effectors, however, interface with both IVA handrails and seat tracks, which do not distribute loads as well as the aforementioned baseplate. The IVA handrails and seat tracks have load and moment limits that R2 must remain below to avoid damaging them. When R2 has only one End Effector attached to a handrail or seat track, any imparted load from R2 motion, or a load imparted to R2 from an outside source, will travel through the robot and into the IVA handrail or seat track.

Multiple methods are used to avoid violating these load limits. First, the load cell in each of the Climbing Legs between the End Effector and the rest of the leg is used to monitor force and moments and shut R2 down if a predetermined threshold is met. Second, R2 is constantly calculating its momentum using a kinematic model and the mass of the various parts of R2. Robonaut 2's brainstem will cease motion of all motors if the momentum limit is met. Momentum control is discussed in detail in Section VI. Finally, unlike the current Torso, the R2 Climbing Legs do not include brakes. This allows these joints to comply with imparted loads to a certain extent.

The fact that the Climbing Legs do not contain brakes is important for another reason: emergency egress of the crewmembers. This is another safety aspect that applies to R2 while mobile, but not while stationary. If R2 is near a hatchway, or climbing through one, and a crewmember needs to get by due to an emergency, $\mathrm{R} 2$ can be moved out of the way manually. If $\mathrm{R} 2$ is in the middle of a motion command at this time, the crewmember can

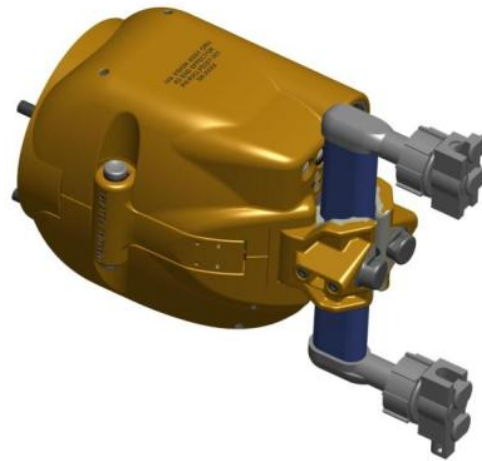

Figure 13. R2 Leg End Effector attached to IVA Handrail.

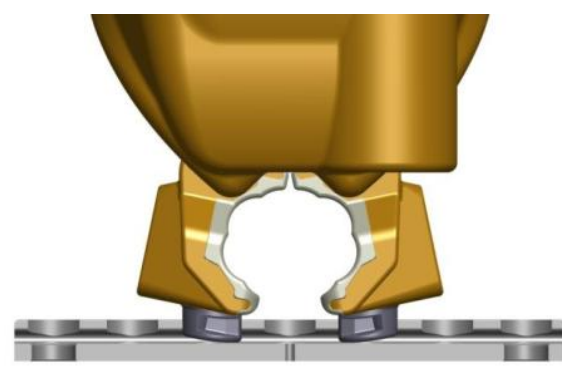

Figure 14. R2 Leg End Effector attached to seat track simply bump R2 deliberately to induce a safe shut-off of the motor power, resulting in Climbing Leg joints that are free to move manually. R2 will sense this bump and shut down due to the monitoring of joint torques and load cell thresholds.

\section{Software Control Upgrades for the IVA Mobile R2}

Hardware and software upgrades are essential for adding mobility to R2's set of capabilities. The core control system requirement for R2 remains the same; the system must be safe for humans to work around. New requirements are added, however, with this new capability. First, the robot must be able to precisely control the location of its End Effector for handrail or seat track grasping while climbing through the ISS. The robot must also be capable of safely accelerating large inertias while maintaining safe and accurate control. R2 will be working around astronauts, so the control system must be able to safely accommodate human interaction just like the current R2 Torso. A crew member must be able to move a limb out of the translation path, but R2 must also be able to smoothly accomplish its climbing goals. Finally, the control system has many joints that must be involved in coordinated control efforts.

These requirements, as well as a desire to upgrade processing hardware, drove a redesign of the R2 control system. The current stationary system runs on the VxWorks operating system in the Constellation control environment. Due to the discontinuation of support for Constellation, its use requires older versions of VxWorks and hardware that would not be capable of running the larger mobility system. After much benchmarking, the Robot Operating System $(\mathrm{ROS})^{*}$, an open-source robotic control environment that runs in Linux, and Core i7 Intel processors were chosen for the new system. ROS provides an interface to Orocos ${ }^{\dagger}$, which is a real-time capable framework and middleware, in which the core control software is developed.

\footnotetext{
* http://www.ros.org/wiki, The Robot Operating System.

${ }^{\dagger}$ http://www.orocos.org, The Orocos Project.
} 
Figure 15 shows a block diagram of the new modelbased robotic dynamic motion control system. The Multi-Loop embedded controller forms the foundation upon which the overall control system is built by ensuring that each joint tracks its commanded trajectory while conforming to desired dynamic performance characteristics and safety thresholds. The custom motor controllers are different than most commercial off-the-shelf versions due to the impedance control loop. The embedded joint torque loop can be directly affected by the coordinated controller by setting joint torque limits as well as sending down a feed-forward torque value (for gravity and inertial compensation). The impedance loop allows the coordinated control processors, the brainstem, to send down position commands at a somewhat low rate while still maintaining excellent tracking performance.

The embedded control layer communicates over a custom protocol to the brainstem through Robonet. This controls data input and output from the brainstem in an efficient way. The joint data is stored in an Application Programming Interface (API) layer that serves as the common interface for the different types of joints for the model-based robotic dynamic motion control system, nicknamed Robodyn.

Robodyn is divided into kinematic and dynamic components. The kinematic components are based on an online trajectory generation and inverse kinematics (IK) solutions. The trajectory generation includes velocity and acceleration limiting to ensure safe control commands. The inverse kinematics calculation ensures that the robot calculates and commands joint positions that will best achieve the cartesian commanding. This differs from the brainstem impedance controller that the stationary R2 currently uses in that actual joint positions are commanded whereas the R2 Mobility control system essentially attaches a virtual spring to the End Effector and "pulls" the arm into position using a Jacobian transpose joint torque calculation. This is important because the extra IK calculations allow the robot to reach its point with more accuracy. Since the robot has many degrees of freedom, the IK solution also allows the control system to use the robot's nullspace more effectively. Robodyn employs a task reconstruction null space optimization solution that optimizes over joint position limits, kinetic energy, and self-collision avoidance as possible.

The dynamic components include gravity and inertial compensation calculations based on a Recursive Newton-Euler Algorithm ${ }^{11}$. This inverse dynamics calculation provides feed-forward torques to the embedded controllers. This compensatory torque is applied in addition to the limited joint torque that is used to achieve the embedded impedance loop. The effect is that small joint torque limits are able to provide responsive and accurate position tracking,

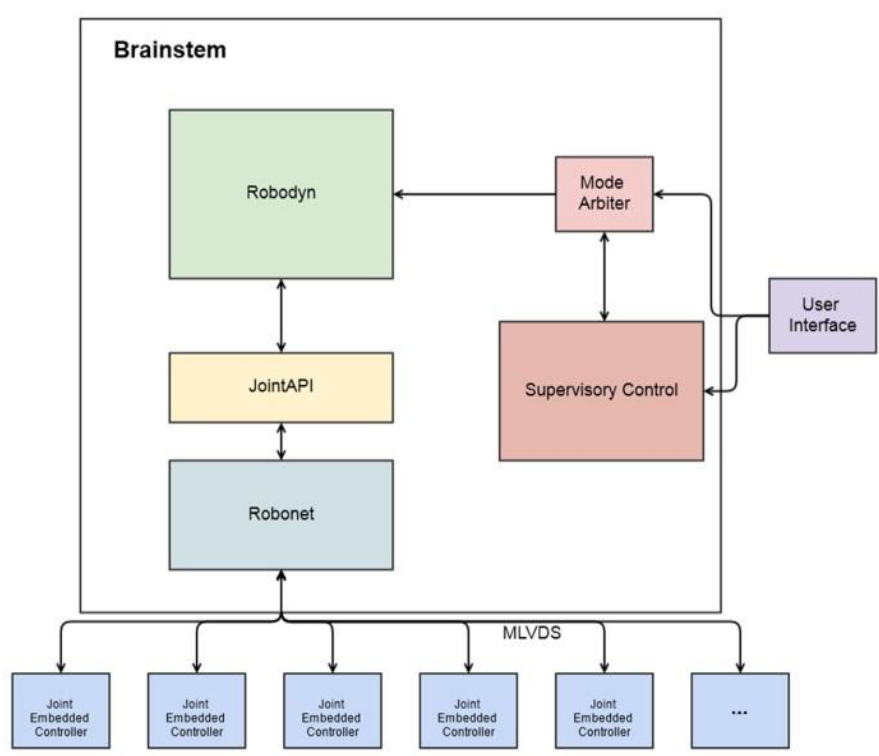

Figure 15. Overall control architecture for Robonaut 2

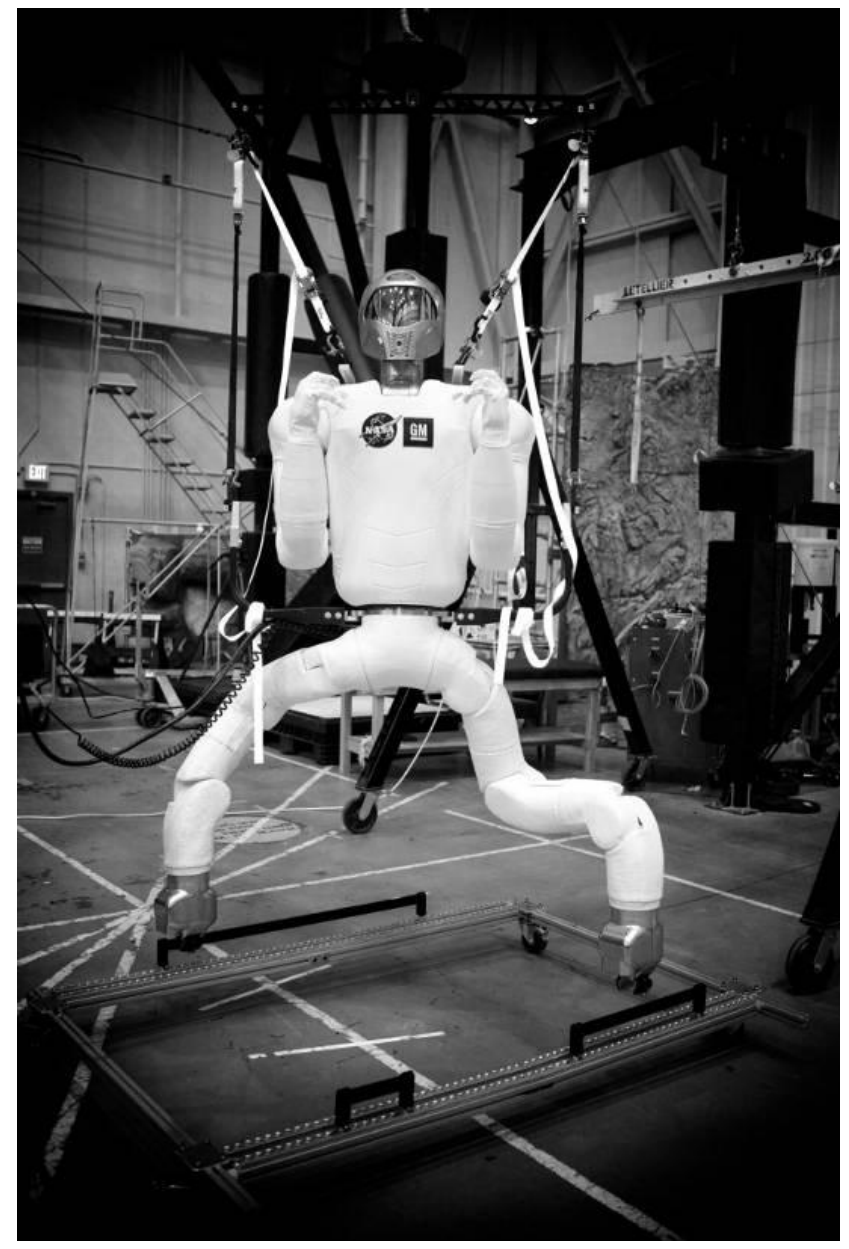

Figure 16. R2 testing mobility algorithms in Active Response Gravity Offload System (ARGOS). 
which adds to the safety of the system. The joints' effective inertias also automatically influence the dynamics of the joint. Impedance loop gains are chosen based on effective inertia, desired natural frequency and damping ratio. This compensates for wide swings in effective inertias seen by the base leg versus the Climbing Leg.

The new control system has been tested by developing many applications to demonstrate its capabilities. Figure 16 shows the R2 Mobility System climbing on an IVA handrail mockup in the NASA-Johnson Space Center's Active Response Gravity Offload System (ARGOS) facility. Figure 17 shows a picture of the R2 Climbing Leg repeatedly drawing a box on a white board to demonstrate its accuracy by going back to the same point many times. Figure 18 shows an instance of inadvertent human interaction. Joint torque limits are kept low, and the kinematic controller components are able to identify the interaction and smoothly null out its current trajectory to ensure that the force on

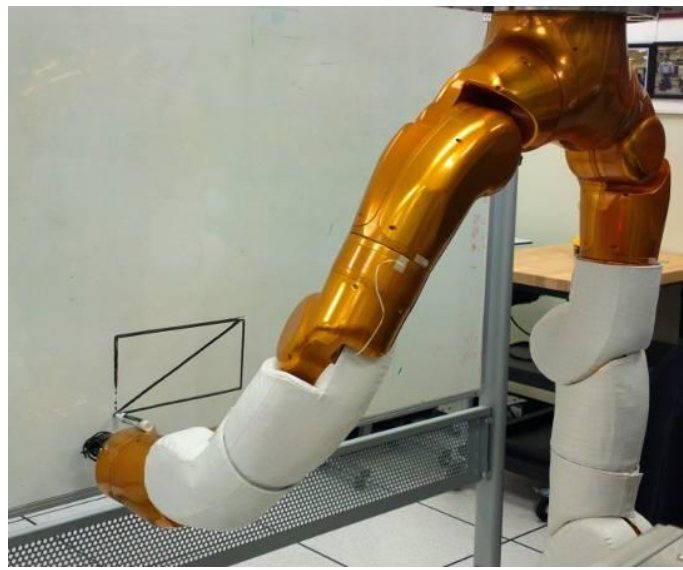

Figure 17. R2 legs performing line drawing test. the human or the environment remains low.

Because of the ISS environment that R2 is designed to be deployed in, there are many safety monitoring requirements to ensure the safety of both the crew and the ISS structure and components. An extension of the Robodyn control system features many monitors and checks that are capable of stopping the robot in several ways, such as removing power and disabling motor controllers. The types of checks that are performed include checking sensor health, force applied, and system momentum. Robodyn includes many controls to mitigate the need to stop the robot for these safety concerns, including limiting trajectory velocities and accelerations, limiting joint torques, and including kinetic energy optimizations in the null space calculations, which penalizes the movement of joints with large effective inertias.

The control architecture shown in Figure 15 also features a supervisory control layer that will house many of the higher-level control and vision capabilities. High level supervisory control functions include an auto-docking capability for handrail grasping. This capability will combine vision algorithms for handrail localization and obstacle determination and avoidance, low-frequency visual servoing for driving the leg into a grasping position, and smart gripper control to ensure good, verified handrail grasps. Other functionalities, including many of the operational tasks that will both contribute to IVA life on board the ISS as well as provide a nearly in situ platform for EVA task development, will also be a part of this supervisory control layer.

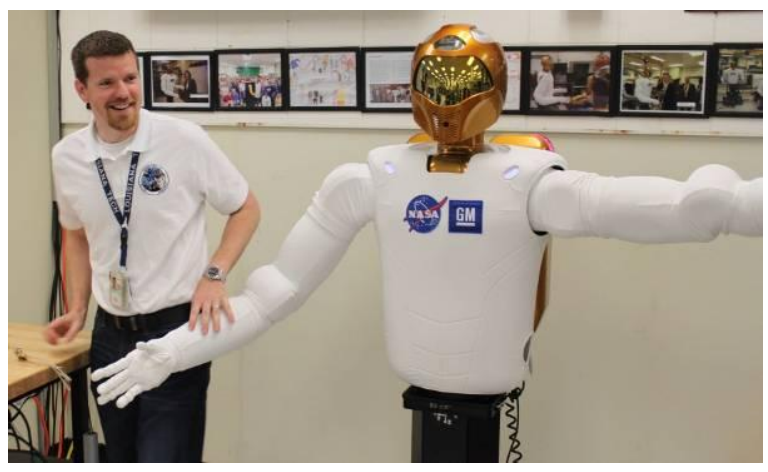

Figure 18. Human interaction with R2.

\section{Future Activities}

\section{A. IVA Mobility Task Objectives}

Once R2 gains Climbing Legs and the Batpack with wireless communication capabilities, the number of potential uses for R2 inside ISS will increase substantially. Other project managers within NASA and JSC have come forward with many task opportunities that the R2 Team is investigating. One of these is IVA handrail cleaning. Like any other habitable environment, the ISS gets dirty and the on-orbit crew members are tasked with keeping it clean. Cleaning handrails is tedious and the crewmembers have many other tasks that their time may be better spent completing. This type of task is precisely why $\mathrm{R} 2$ can be a valuable addition to the IVA crewmembers support team. Once mobile, R2 can translate within the US Laboratory and clean IVA handrails, as well as perform other general housekeeping tasks such as stowing loose items. At first, R2 will only be capable of translating throughout the US Lab, but future goals include gaining access to the Japanese Space Agency (JAXA) Japanese Experiment Module (JEM) as well as the European Space Agency (ESA) Columbus Module. These modules also have IVA handrails and may have other tasks that are suitable for R2. 
As previously discussed, R2 has also demonstrated the potential to be a valuable tool for environmental monitoring for the ECLSS team by its use of the Velocicalc. The R2 team plans on continuing to use this tool, and once R2 is mobile, many other air vents will be accessible for R2 to assess. Other ECLSS sensors and monitors, such as $\mathrm{O} 2$ and $\mathrm{CO} 2$ sensors, may be available for $\mathrm{R} 2$ to inspect and monitor to possibly free up on-orbit crewmember time for other tasks.

Emergency scenarios are another area that R2 may provide a great benefit for on-orbit. Emergency situations on ISS may require the on-orbit crewmembers to close module hatches to contain fires or contain depressurization events isolated to only part of ISS. Once these emergency situations have been assessed and controlled, R2 provides a perfect tool to enter the potentially dangerous area and do a dexterous inspection while providing real-time video to controllers on the ground.

Another task that R2 is already training for on the ground is using the Radio Frequency Identification Device (RFID). Keeping track of all the items on ISS is a huge and challenging task. The ISS program uses both barcodes and more recently RFID tags to track and organize items on-orbit. R2 has already practiced using an RFID scanning device on the ground and it has completed its first trial on-orbit in preparation to help the crew with this task.

\section{B. Future upgrades for EVA}

R2's ultimate goal, as has been discussed previously, is to aid spacewalkers and the ISS program with EVA tasks. IVA mobility is an important step toward this goal to demonstrate both the climbing capability, battery power and wireless data transmission. Without these capabilities an EVA R2 would be very restricted in the EVA environment.

The Climbing Legs that are being used in the IVA environment are also planned for use during R2 EVA operations. All of the materials in the Climbing Legs are EVA compatible and some thermal vacuum testing has been performed on the actuators and motors drivers. The Climbing Legs are not being certified for EVA use until a later date, but an identical set of Climbing Legs will be available on the ground and will be used for certification by similarity at a later date when R2 is ready to enter the EV environment. The Batpack, R2 Torso and a minimal set of sensor related components in the end effectors will need to be replaced for R2 to be compatible in the EV environment. Both use fans to help cool electronics and have various materials that are not EVA compatible. The main challenge for both the Batpack and the Torso will be heat sinking all of the electronics to sufficiently radiate heat to avoid overheating. Heaters can be installed to help alleviate the cold temperature situations, but active cooling loops add complexity and current design concepts do not require them. The EV environment varies between very cold and very hot temperatures. The temperatures experienced while EVA depend greatly on the angle between the sun's vector and the orbital plane of ISS, called the beta angle. The ISS program and ground controllers try to keep a beta angle of approximately 0 degrees, but some situations require larger beta angles that result in more extreme temperatures. A combination of heaters, thermal heat sinking and operational control and restrictions will be used to handle the large temperature swings that the EV environment can pose.

Another important aspect to an R2 in the EV environment is determining a suitable home base location. Translating R2 in and out of the ISS each time the R2 Team wants to perform an EVA operation will use valuable consumables on ISS. To avoid using these consumables, analysis is being performed to determine an acceptable location for R2 to reside permanently in the EV environment. Multiple locations are already being investigated. The location that is eventually chosen will require a power connection for R2 to charge its Batpack and access to a wireless network for data transmissions. Desired, but not required, are a benign thermal environment and micrometeoroid orbital debris (MMOD) and atomic oxygen (AO) protection.

\section{Future EVA Tasks}

Robotic devices are already in use in the EV environment. These include the previously discussed SSRMS and SPDM robotic systems. Both provide great benefit to the ISS program and aid in EVA operations. An EVA mobile R2 will augment the capabilities of these robots by providing a dexterous humanoid robot capable of performing tasks that thus far have only been performed by human crewmembers.

Many of the tasks that R2 is well suited for in the EVA environment are inspection and contingency operations. R2 provides a mobile device, along with effectors that can both

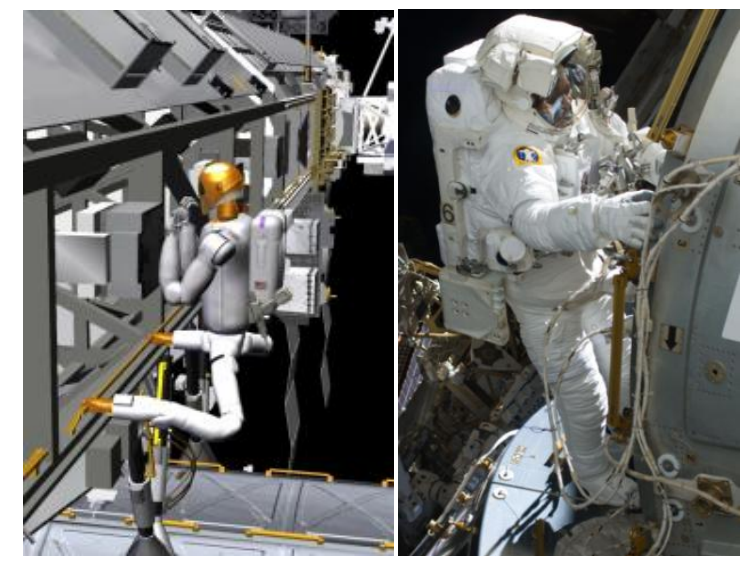

Figure 19. R2 EVA unit and EV crewmember 
climb and move soft goods out of the way, and also obtain images or video of the ISS. Situations on the ISS arise relatively often where quality images or video of areas in question would be of great benefit. Examples of this are ammonia pump leaks or Solar Alpha Rotary Joint (SARJ) debris issues. Both of these instances occurred on ISS in the past and required EV crewmembers to go out to these devices to inspect them. Performing EVAs is very expensive and time consuming. An EVA capable R2 can make human EVAs more efficient by assisting the crew with necessary inspections and also provide worksite setup and teardown capabilities by taking tools to and from these worksites.

Another task that R2 can perform, which is related to

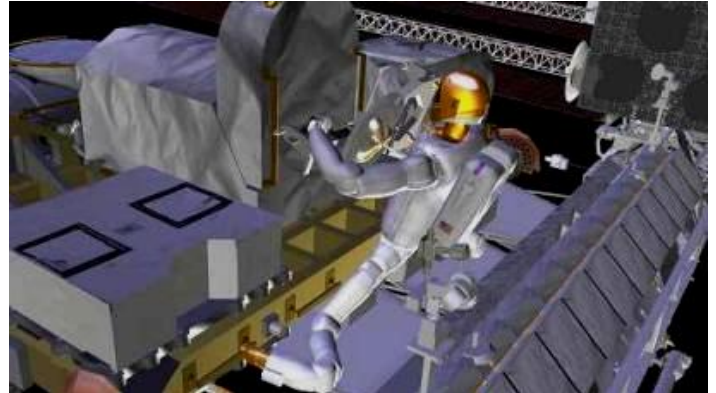

Figure 20. R2 performing the Adjustable Grapple Bar (AGB) reloaction worksite setup and teardown, is moving and installing/un-installing Articulating Portable Foot Restraints (APFRs). These devices are used by EV crewmembers to help keep themselves stable while performing tasks at an EV worksite. These devices are time consuming to move by EV crewmembers and reduce the precious EVA time that is allowed based on the amount of consumables the EMU can carry. The Adjustable Grapple Bar (AGB) is another item that R2 could pre-locate in preparation for a crew activity. This tool is specifically used in the ammonia tank removal and replacement $(\mathrm{R} \& \mathrm{R})$ procedure that was previously discussed. A dexterous manipulator, like the $\mathrm{R} 2$ hand, is required to move this tool because it requires the use of the Pistol Grip Tool (PGT) to relocate. ${ }^{10} \mathrm{R} 2$ could provide this capability to save EV crew time in the face of an urgent situation like an ammonia pump failure.

\section{Conclusion}

As of the writing of this paper, the ISS has been in orbit around Earth for over 5000 days. This is an incredible feat considering the harsh environment that the ISS has to withstand every second of every day. The ground personnel and on-orbit crewmembers who keep the ISS in good health have performed with near flawless precision in the face of many challenges. R2 is another tool that these engineers and astronauts will use to help maintain ISS and perform valuable science. In both the IVA and EVA environments, a mobile R2 can be used for both monotonous tasks saving valuable crew time, as well as contingency situations where it can assist the crew by taking on risks during inspections and repairs.

\section{References}

${ }^{1}$ Stieber, M., Trudel, C., Hunter, D., "Robotic systems for the International Space Station," Proceedings of the IEEE International Conference on Robotics and Automation, Albuquerque, New Mexico, 1997, pp. 3068-3073.

${ }^{2}$ Hirzinger, G., Brunner, B., Dietrich, J., Heindl, J., "ROTEX-The First Remotely Control Robot in Space," Proceedings of the IEEE International Conference on Robotics and Automation, San Diego, California, 1994, pp. $2604-2611$.

${ }^{3}$ Oda, M., et al., "ETS-VII: Achievements, Troubles, and Future," Proceedings of the 6th International Symposium on Artificial Intelligence and Robotics \& Automation in Space: I-SAIRAS 2001. Montreal, Canada 2001.

${ }^{4}$ Fredrickson, S. E., Lockhart, P. S., and Wagenknecht, J. D., "Autonomous Extravehicular Robotic Camera (AERCam) for Remote Viewing," Proceedings of the AIAA International Space Station Service Vehicles Conference, Houston, Texas, 1999.

${ }^{5}$ Albu-Schaffer, A., et al. "ROKVISS- Robotics Component Verification on ISS Current Experimental Results on Parameter Identification," Proceedings of the IEEE International Conference on Robotics and Automation, Orlando, Florida, 2006, pp. 3879 -3885 .

${ }^{6}$ Hoffman, M., "Robotic Refueling Project of NASA Conducted First Test at ISS," Science World Report [online report], URL:http://www.scienceworldreport.com/articles/4858/20130209/robotic-refueling-project-nasa-conducted-firsttest.htm [cited 09 Feb 2013].

${ }^{7}$ Diftler, M. A., et al., "Robonaut 2- The First Humanoid Robot in Space," Proceedings of the IEEE International Conference on Robotics and Automation, Shanghai, China, 2011, pp. 2178 - 2183.

${ }^{8}$ Etsinger-Gonzales, A. and Weber, J., "Domo: A Force Sensing Humanoid Robot for Manipulation Research," Proceedings of IEEE/RAS Intl. Conf. on Humanoid Robots, 2004, pp. 273-291.

${ }^{9}$ Bridgwater, L. B., et al., "The Robonaut 2 hand - designed to do work with tools," Proceedings of the IEEE International Conference on Robotics and Automation, St. Paul, Minnesota, 2012, pp. 3425-3430.

${ }^{10}$ Diftler, M.A., et al., "Robonaut 2 - Initial Activities On-Board the ISS," Proceedings of the IEEE Aerospace Conference, Big Sky, Montana, 2012, pp. 1-12.

${ }^{11}$ Featherstone, R., "Rigid Body Dynamics Algorithms," Springer, 2008. 